\title{
Three-dimensional optical transfer of rotating beams
}

\author{
Meir R. Hatzvi* and Yoav Y. Schechner \\ Department of Electrical Engineering, Technion-Israel Institute of Technology, Haifa 32000, Israel \\ ${ }^{*}$ Corresponding author: hatzvi@tx.technion.ac.il
}

Received March 12, 2012; revised June 7, 2012; accepted June 7, 2012;

posted June 14, 2012 (Doc. ID 164471); published July 26, 2012

\begin{abstract}
Systems in which the point spread function (PSF) is a rotating beam have increasing use in three-dimensional (3D) microscopy and depth estimation. We analyze in several ways the 3D optical transfer function (OTF) of Gauss Laguerre modes and rotating beams. This is based on analysis of 3D OTFs of general aperture functions. Consequently, we suggest a criterion for depth resolution based on an effective cutoff of the axial frequency response. This criterion can be used to optimize PSFs explicitly and directly, to maximize axial resolution. (C) 2012 Optical Society of America
\end{abstract}

OCIS codes: $\quad 070.2580,110.1220,110.4850$.

Optical volumetric microscopy is widely used in biology and medicine to study cells and tissues. Microscopy seeks increased spatial resolution. For volumetric objects, there is need for high depth $(z)$ resolution, in particular. There are many three-dimensional (3D) microscopy methods [1]. Each method has advantages and disadvantages. Common methods are based on optical sectioning: a 3D data cube is acquired, and then $3 \mathrm{D}$ deconvolution recovers the object. This Letter is motivated by wide field optical sectioning. Here a set of images is acquired, each at different focus setting. Relative to competing methods, advantages of this modality are simplicity, speed, and functioning with or without fluorescence or acoustic probes. However, its depth resolution has so far been inferior to competing methods. This Letter extends the analysis of linear optical 3D transfer, in the context of depth resolution. Such analysis can yield systems having improved depth resolution while maintaining the advantages of existing imaging modalities.

Linear incoherent volumetric imaging is characterized by a 3D intensity point spread function (PSF). Similarly, the intensity across the 3D exit domain of an imaging system is characterized. Linear systems theory (3D frequencies) expresses the optical transfer. Axial resolution depends on the system's axial cutoff frequency. By maximizing this resolution, points at different axial coordinates can be better discriminated.

Let $\mathbf{x} \equiv(x, y)$ be the lateral position. The 3D PSF is $I(\mathbf{x}, z)$. Let $\mathbf{f} \equiv\left(f_{x}, f_{y}\right)$ and $f_{z}$ be the spatial frequency variables corresponding to $\mathbf{x}$ and $z$, respectively. The lateral spatial frequency domain can be defined by an aperture plane, behind a lens. The aperture domain has area units. We prefer to use spatial frequency units satisfying $\mathbf{f}=\frac{\mathbf{x}}{\lambda F}$, where $F$ is the focal length of the lens and $\lambda$ is the wavelength. The aperture function is $\hat{p}(\mathbf{f})$ as illustrated in Fig. 1. The Fourier transform of the intensity 3D PSF is the 3D optical transfer function (OTF) of the system. Following [2], in the paraxial approximation, the $3 \mathrm{D}$ OTF is

$$
\hat{I}\left(\mathbf{f}, f_{z}\right)=\iint_{\mathbf{v}} \hat{p}(\mathbf{v}+\mathbf{f} / 2) \hat{p}^{*}(\mathbf{v}-\mathbf{f} / 2) \delta\left[\lambda(\mathbf{v} \cdot \mathbf{f})+f_{z}\right] \mathrm{d} \mathbf{v} .
$$

Here $\mathbf{v} \equiv\left(v_{x}, v_{y}\right)$ are integration variables, $\delta$ is the Dirac delta function, and $*$ denotes complex conjugation. To simplify calculations, we exploit a property of $\delta$ functions [3]. For a function $h\left(v_{x}\right)$ whose root is $v_{x 0}$,

$$
\delta\left[h\left(v_{x}\right)\right]=\delta\left(v_{x}-v_{x 0}\right) /\left|h^{\prime}\left(v_{x 0}\right)\right|,
$$

where $h^{\prime}\left(v_{x}\right)$ is the derivative of $h\left(v_{x}\right)$. Therefore,

$$
\delta\left[\lambda(\mathbf{v} \cdot \mathbf{f})+f_{z}\right]=\left(1 /\left|\lambda f_{x}\right|\right) \delta\left[v_{x}-\left(f_{z}+\lambda v_{y} f_{y}\right) / \lambda f_{x}\right] .
$$

By integrating over $d v_{x}$, Eq. (1) becomes a onedimensional integral expression:

$$
\begin{aligned}
\hat{I}\left(\mathbf{f}, f_{z}\right)= & \frac{1}{\left|\lambda f_{x}\right|} \int \hat{p}\left[-\frac{f_{z}+\lambda v_{y} f_{y}}{\lambda f_{x}}+\frac{f_{x}}{2}, v_{y}+\frac{f_{y}}{2}\right] \\
& \times \hat{p}^{*}\left[-\frac{f_{z}+\lambda v_{y} f_{y}}{\lambda f_{x}}-\frac{f_{x}}{2}, v_{y}-\frac{f_{y}}{2}\right] \mathrm{d} v_{y} .
\end{aligned}
$$

The 3D OTF $\hat{I}\left(\mathbf{f}, f_{z}\right)$ can also be derived directly from the intensity 3D PSF of the system:

$$
\hat{I}\left(\mathbf{f}, f_{z}\right)=\iiint I(\mathbf{x}, z) \exp \left[-i 2 \pi\left(\mathbf{x} \cdot \mathbf{f}+z f_{z}\right)\right] \mathrm{d} \mathbf{x} \mathrm{d} z .
$$

High $f_{z}$ frequencies correspond to small axial details. The higher the frequencies that the system passes, the better the axial resolution. Characterizing the $f_{z}$ response thus indicates limits of depth reconstruction. To extract the frequency response along $f_{z}$, we integrate the signal energy in Eq. (4) or Eq. (5) over f:

$$
E\left(f_{z}\right)=\iint_{\mathbf{f}}\left|\hat{I}\left(\mathbf{f}, f_{z}\right)\right|^{2} \mathbf{d f}
$$

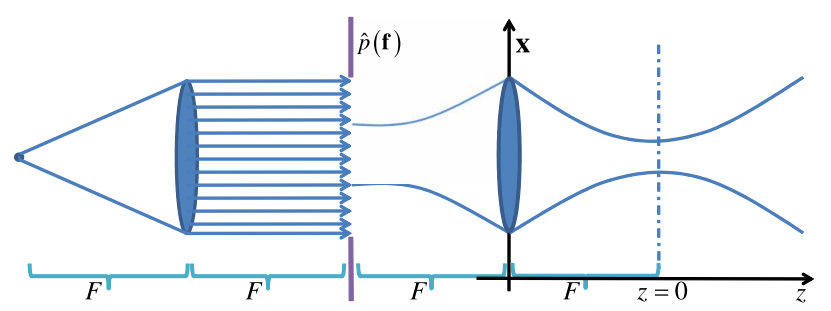

Fig. 1. (Color online) Imaging system with an aperture function $\hat{p}$ in the Fourier plane. $z$ is the axial coordinate. 
Consider rotating beams [늠 $\underline{6}$ ] as PSFs. Then, lateral cross sections taken at different axial locations differ from each other only by a rigid rotation and scale (Fig. 2 ). Formally, $\forall\left\{z_{1}, z_{2}\right\}, \exists\{s, \Delta \varphi\}$ such that

$$
I\left(\|\mathbf{x}\|, \varphi, z_{1}\right)=I\left(\|\mathbf{x}\| / s, \varphi+\Delta \varphi, z_{2}\right),
$$

where in polar coordinates $\mathbf{x}=(\|\mathbf{x}\|, \varphi)$. Here $\varphi$ is the azimuthal angle. Rotating PSFs [4] have been used in microscopy to estimate depth $[\underline{5}, \underline{7}, \overline{8}]$. Paraxial rotating PSFs can be easily expressed using the Gauss-Laguerre (GL) basis. A GL function is an eigenmode of paraxial wave propagation. In polar coordinates, $\mathbf{f}=(\|\mathbf{f}\|, \varphi)$. Let $\sigma$ be the standard deviation (STD) of a Gaussian in the spatial frequency domain. Define $\tilde{\mathbf{f}} \equiv \mathbf{f} / \sigma$, and $\tilde{f}_{z} \equiv f_{z} / \sigma$ as the spatial frequencies scaled by the Gaussian width. The Gaussian $g(\|\tilde{\mathbf{f}}\|)=\exp \left(-\|\tilde{\mathbf{f}}\|^{2} / 2\right)$ is common to all GL modes. Let $L_{(n-|m|) / 2}^{|m|}$ be the generalized Laguerre polynomials. The integers $n, m$ satisfy $n=\{|m|,|m|+2$, $|m|+4, \ldots\}$. Similar to []] , define

$$
R_{n, m}(\|\tilde{\mathbf{f}}\|)=\|\tilde{\mathbf{f}}\|^{|m|} L_{(n-|m|) / 2}^{|m|}\left(\|\tilde{\mathbf{f}}\|^{2}\right) .
$$

Then, a GL basis function has the form

$$
\langle\mathbf{f} \mid n, m\rangle_{\sigma}=u_{n, m}(\mathbf{f})=g(\|\tilde{\mathbf{f}}\|) R_{n, m}(\|\tilde{\mathbf{f}}\|) \Phi_{m}(\varphi),
$$

where $\Phi_{m}(\varphi)=\exp (\operatorname{im} \varphi)$. Any aperture function $\hat{p}$ is a linear combination of GL basis functions:

$$
\hat{p}(\mathbf{f})=\sum_{n, m} \alpha_{n, m}\langle\mathbf{f} \mid n, m\rangle_{\sigma}
$$

where $\alpha_{n, m}$ is the coefficient of mode $|n, m\rangle$. We use $\sigma$ as a parameter for the beams.

Certain superpositions of GL modes [4] yield rotating PSFs. This occurs when $\alpha_{n, m}=0$, other than for modes $j$ satisfying $\frac{n_{j+1}-n_{j}}{m_{j+1}-m_{j}}=$ const. Figure $\underline{3}$ shows slices of $\hat{I}\left(\mathbf{f}, f_{z}\right)$ corresponding to a rotating PSF (denoted Beam 1). This is a superposition [5] where $\alpha_{1,1}=\alpha_{5,3}=\alpha_{9,5}=\alpha_{13,7}=$ $\alpha_{17,9}=1$ and $\alpha_{n, m}=0$ otherwise.

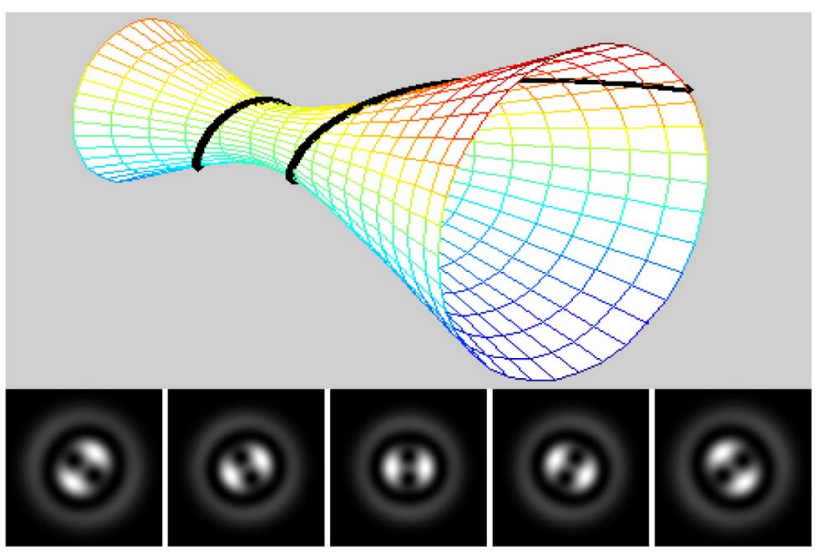

Fig. 2. (Color online) (top) Rotating beam. The beam propagates along the axial direction while (bottom) its lateral cross sections change only by scale and rigid rotation.

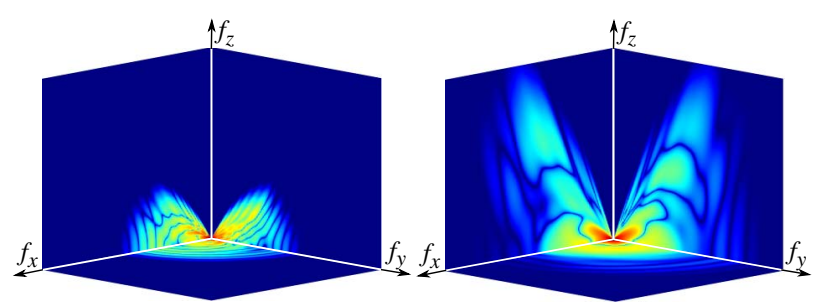

Fig. 3. (Color online) 3D OTF $\left[\log \left|\hat{I}\left(\mathbf{f}, f_{z}\right)\right|\right]$ of (left) Beam 1 and (right) optimized Beam 1. The energy for high $f_{z}$ is significantly higher after the optimization.

To fairly compare different 3D PSFs, we normalize them in two ways. First, no matter what the superposition is, the total energy in any lateral cross section is

$$
\iint|\hat{p}(\mathbf{f})|^{2} \mathbf{d} \mathbf{f}=1 .
$$

Thus, different aperture functions do not induce different optical energies in the 3D PSF. All 3D PSFs should have the same effective width in the aperture plane. We ensure that by using a second normalization. For the aperture intensity distribution $|\hat{p}(\mathbf{f})|^{2}$, the effective width of mode $|0,0\rangle$ is $\sigma / \sqrt{2}$. In higher-order modes, the width of $|\hat{p}(\mathbf{f})|^{2}$ differs if $\sigma$ is constant. Therefore, $\sigma$ must change for superpositions that contain significant energy in highorder modes. The covariance matrix of $\hat{p}(\mathbf{f})$ is

$$
\Sigma=\iint(\mathbf{f}-\overline{\mathbf{f}})^{T}(\mathbf{f}-\overline{\mathbf{f}})|\hat{p}(\mathbf{f})|^{2} \mathrm{~d} \mathbf{f} .
$$

Here $T$ denotes transposition and $\overline{\mathbf{f}}=\iint \mathbf{f}|\hat{p}(\mathbf{f})|^{2} \mathrm{df}$. For symmetric PSFs, $\overline{\mathbf{f}}=0$. Let the eigenvalues of $\Sigma$ be $\nu_{1}<\nu_{2}$. The effective width of $|\hat{p}(\mathbf{f})|^{2}$ is $\sqrt{\nu_{2}}$. It is set to be the same for all beams, by adapting $\sigma$.

We now study the highest axial frequency that a system passes (an effective cutoff). Consider frequency $f_{z}$ effectively passed if $E\left(f_{z}\right)$ is larger than a threshold $\tau$. In this Letter, we chose $\tau=0.05$. The effective frequency cutoff can be a criterion for beam optimization. Let us seek coefficients that yield the widest $f_{z}$ response. Let $A=$ $\left\{\alpha_{n, m}\right\}$ be the set of the coefficients of a superposition of GL modes. Therefore,

$$
\hat{A}=\operatorname{argmax}_{A}\left\{\operatorname{cutoff} E\left(f_{z}\right)\right\}
$$

is a set defining an optimized 3D PSF. To demonstrate this, we perform optimization. Starting from initial superposition of several modes, the coefficients $\alpha_{n, m}$ of these modes vary [9] to optimize Eq. (13) locally in coefficient space. We allow $\alpha_{n, m}$ to be complex. This yields better 3D PSFs than using real-valued coefficients, thanks to increased degrees of freedom.

Numerical optimization involves a large number of iterations. To speed them, Eq. (5) is calculated using fast Fourier transform (FFT) over a discrete grid. Using FFT involves some numerical artifacts and singularity issues. Nevertheless, when FFT is properly done, we receive results consistent with Eq. (4) for most spatial frequencies. Once optimization converged, the 3D OTF was finally calculated using the more accurate Eq. (4). 

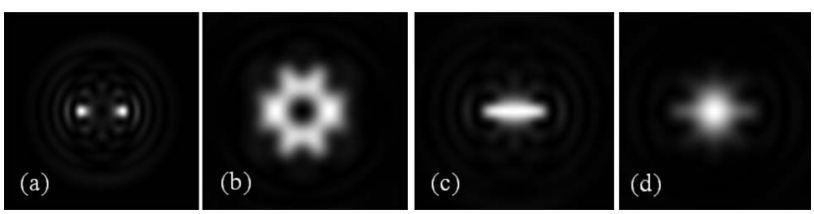

Fig. 4. Intensity lateral cross sections (contrast stretched for display) of Beam 1 (a) before and (b) after optimization and Beam 2 (c) before and (d) after optimization.

Figures 4(a) and 4(c) show intensity distributions of two rotating PSFs: one initialized by Beam 1, the other initialized by $\alpha_{0,0}=\alpha_{4,2}=\alpha_{8,4}=\alpha_{12,6}=\alpha_{16,8}=1$, and $\alpha_{n, m}=0$ otherwise (Beam 2). Figures 4(b) and 4(d) show the intensity distributions after optimizing the respective nonzero coefficients of each beam, under the said normalizations.

To compute different beams, we use as reference a Gaussian aperture for which the intensity STD is $\sigma_{0}=\left.\sqrt{\nu_{2}}\right|_{\text {Gaussian }}=1 /(6 \sqrt{2} \lambda)$. This sets a constant scale for $f_{z}$ across all apertures. Define $f_{z}^{\text {norm }}=f_{z} / \sigma_{0}$.

Figure 5 shows simulated comparisons of standard apertures (clear and Gaussian) and apertures of some rotating beams. The plots were calculated using Eq. (4). A clear aperture has a sharp $f_{z}$ cutoff. This cutoff is induced by the aperture's sharp cutoff in $\|\tilde{\mathbf{f}}\|$, via the missing cone of frequencies [10]. The other apertures are not truncated yet have the same effective aperture width.

A system having a rotating PSF can be implemented by a mask that encodes $\hat{p}$ in the back focal (Fourier) plane of an objective lens (Fig. 1) [4,5]. This mask, also called aperture function, can be decomposed as a superposition of GL basis functions. Beam 1 can also be approximated in a practical device using a phase-modulated clear aperture [7]. Reference [10] found this approximate 3D PSF to be prone to the same missing cone limitations as a standard aperture's 3D PSF. The rotating PSFs in Fig. $\underline{5}$ are not approximate (in the paraxial regime) and are based on nontruncated apertures. In low $f_{z}$, the response of Beam 1 is lower, but it overtakes the clear aperture at higher $f_{z}$. According to the effective cutoff criterion, Beam 1 has better axial resolution than a clear aperture but worse than a Gaussian that has the same effective lateral width. 3D OTF is indicative to volumetric imaging, where the object is dense and thick in $3 \mathrm{D}$, requiring $3 \mathrm{D}$ deconvolution for recovery. In such complex objects, Beam 1 does not appear to be advantageous over a Gaussian having the same energy and scale normalizations, under linear systems analysis.

Nevertheless, Fig. 5 shows that rotating PSFs can have a significantly higher $f_{z}$ cutoff than Beam 1 and Gaussian beams. This indicates significant potential for enhancement of axial resolution. These enhanced PSFs are automatically designed by optimization, which explicitly seeks to maximize the $f_{z}$ cutoff. This framework can be extended to other 3D PSFs (other apertures), not only

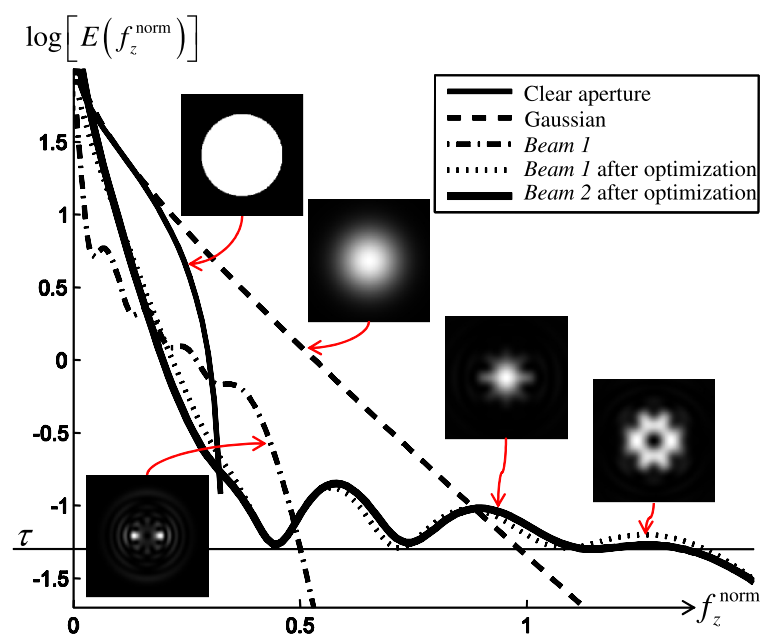

Fig. 5. (Color online) $\log \left[E\left(f_{z}^{\text {norm }}\right)\right]$ for clear and Gaussian apertures, Beam 1, and Beam 1 and Beam 2 post optimization.

rotating beams. Specifically, practical phase-only truncated masks can be directly optimized [11] to increase the $f_{z}$ cutoff. Other resolution measures can be used for optimizing 3D PSFs. In addition, a more practical analysis can be executed considering finite apertures and aberrations.

We thank Joseph Shamir for useful discussions. This work was supported by the R. L. Kohns Eye Research Fund. It was conducted in the Ollendorff Minerva Center. Minerva is funded through the BMBF. Y. Y. S. is a Landau Fellow supported by the Taub Foundation.

\section{References}

1. V. Ntziachristos, Nat. Methods 7, 603 (2010).

2. N. Streibl, Optik 66, 341 (1984).

3. I. M. Gel'fand and G. E. Shilov, Generalized Functions (Academic, 1964).

4. Y. Y. Schechner, R. Piestun, and J. Shamir, Phys. Rev. E 54, R50 (1996).

5. A. Greengard, Y. Y. Schechner, and R. Piestun, Opt. Lett. 31, 181 (2006).

6. R. Horstmeyer, S. B. Oh, R. Raskar, and H. Gao, in Digital Holography and Three-Dimensional Imaging, OSA Technical Digest (CD) (Optical Society of America, 2011), paper DWC23.

7. S. R. P. Pavani, M. A. Thompson, J. S. Biteen, S. J. Lord, N. Liu, R. J. Twieg, R. Piestun, and W. E. Moerner, Proc. Natl. Acad. Sci. USA 106, 2995 (2009).

8. M. D. Lew, S. F. Lee, M. Badieirostami, and W. E. Moerner, Opt. Lett. 36, 202 (2011).

9. S. Barwick, Appl. Opt. 47, 5893 (2008).

10. S. Ghosh and C. Preza, in Computational Optical Sensing and Imaging, OSA Technical Digest (CD) (Optical Society of America, 2011), paper CTuB3.

11. M. E. Testorf and M. A. Fiddy, J. Opt. Soc. Am. A 18, 2908 (2001). 